\title{
RESONANT COOPER-PAIR TUNNELING: COUNTING STATISTICS AND FREQUENCY-DEPENDENT CURRENT NOISE
}

\author{
A.A. CLERK \\ Departments of Applied Physics and Physics \\ Yale University, PO Box 208284, New Haven, CT 06520-8284
}

\begin{abstract}
We discuss the counting statistics and current noise associated with the double Josephson quasiparticle resonance point in a superconducting single electron transistor. The counting statistics are in general phase-dependent, despite the fact that the average current has no dependence on phase. Focusing on parameter regimes where the counting statistics have no phasedependence, we use a general relation first derived by MacDonald in 1948 to obtain the full frequency-dependent shot noise directly from the counting statistics, without any further approximations. We comment on problems posed by the phase-dependence of the counting statistics for the finitefrequency noise.
\end{abstract}

\section{Introduction}

Resonant Cooper-pair tunneling, also known as Josephson quasiparticle tunneling, refers to transport cycles in superconducting single-electron transistors (SSET's) which involve the transfer of both Cooper pairs and quasiparticles 1, 2, 3. They have recently been the subject of renewed attention, both because of their unusual noise properties [4, 5, 6] and because of their utility in measuring the state of a charge superconducting qubit [7, 8. In terms of noise properties, it has been shown that charge fluctuations associated with these processes can induce a population inversion in a coupled two-level system (i.e in terms of its charge noise, the transistor effectively has a negative temperature) [5, 6. The shot-noise in the current through the transistor was also found to have remarkable properties [4, 5]. By tuning the strength of the Cooper pair tunneling relative to the quasiparticle tunneling, one could effectively tune the Fano factor determining the zero-frequency 
shot noise. It was possible to achieve a Fano factor greater than one, which was interpreted as a consequence of the effective charge associated with the transport cycle being greater than one. Perhaps more surprisingly, it was possible to reduce the Fano factor below 1/2, behaviour that was not fully explained. The finite frequency current noise also showed interesting behaviour 4] - in the regime where the Cooper-pair tunneling dominated the quasiparticle tunneling, a coherent peak in the current noise was predicted at the Josephson energy.

In this paper, we look specifically at the statistics of transferred charge and current noise associated with the double Josephson quasiparticle (DJQP) process in a SSET, a process which allows for near quantum-limited measurement [5], and which has been used in a recent experiment [8. We pay careful consideration to the fact that the breaking of gauge invariance by superconductivity can make the interpretation of counting statistics more subtle than in the normal-metal case [10, 12, 13. One obtains phasedependent counting statistics here despite the fact that transport is insensitive to the overall phase difference between the two superconducting reservoirs. We identify two regimes in which there is no ambiguity in defining counting statistics, and proceed to use them to directly calculate the frequency-dependent shot noise, without any further approximations. This is in contrast to previous treatments both of normal and superconducting single electron transistors 14, 15, 4, where an additional Markov approximation (beyond what is needed for the counting statistics) is made to obtain the frequency-dependent current noise. The direct connection between counting statistics and frequency-dependent current noise we employ here allows one to give a simple interpretation of the latter. In particular, coherent peaks in the shot noise appearing at the Josephson energy can be directly tied to the time dependence of the counting statistics. We also suggest that the suppression of the Fano factor below 1/2 is related to the coherence of Cooper-pair tunneling.

\section{Description and Model for Josephson Quasiparticle Tunneling}

A SSET consists of a superconducting, Coulomb-blockaded island which is attached via tunnel junctions to two superconducting electrodes (Fig. 1). The SSET Hamiltonian $\mathcal{H}_{S}=H_{K}+H_{C}+H_{V}+H_{T}$ has a term $H_{K}$ describing the kinetic energy of source, drain and central island electrons (i.e. three bulk BCS Hamiltonians), a term $H_{V}$ which describes the work done by the voltage sources, and a tunneling term $H_{T}$. The charging interaction is $H_{C}=E_{C}(n-\mathcal{N})^{2}$, where $E_{C}$ is the SSET capacitive charging energy, $n$ is the number of electrons on the central island, and $\mathcal{N}$ is the dimensionless 


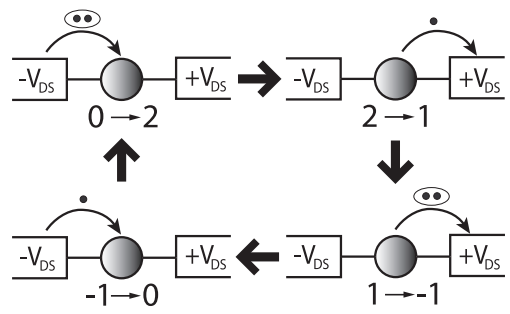

Figure 1. Schematic showing the four steps of the double Josephson quasiparticle process which can occur in a superconducting single-electron transistor. Circles represent the central island of the SSET, while the rectangles are the electrodes. Numbers indicate the charge of the SSET island.

gate voltage applied to the island. We consider a SSET with identical tunnel junctions, whose dimensionless conductance $g$ satisfies $g /(2 \pi) \ll 1$.

The DJQP process occurs when both the transistor gate voltage $\mathcal{N}$ and drain-source voltage $2 V_{D S}$ are tuned such that two Cooper-pair tunneling transitions, one in each junction, are resonant. We label these transitions as $n=0 \rightarrow 2$ in the left junction, and $n=1 \rightarrow-1$ in the right junction (see Fig. 11). The double resonance point for these transitions occurs at $e V_{D S}=E_{C S}$ and $\mathcal{N}_{S}=1 / 2$. In addition, $E_{C} / \Delta$ (where $\Delta$ is the superconducting gap of the SSET) must be chosen so that the quasiparticle transitions linking the two Cooper pair resonances are energetically allowed (i.e. $n=2 \rightarrow 1$ and $n=-1 \rightarrow 0$ ), whereas unwanted transitions which would interrupt the cycle (i.e. $n=0 \rightarrow 1$ ) are not. We take $E_{C}=\Delta$ to satisfy these conditions; this corresponds to the experiment of Ref. [8].

Assuming that all the above conditions are met, and that $T \ll E_{C}$, transport through the SSET will be dominated by the DJQP process sketched in Fig. 1] The standard theoretical description of this process [2] is obtained by looking at the dynamics of the reduced density matrix $\rho$ describing the charge $n$ of the central SSET island. Here, only four charge states are important; further, off-diagonal terms need only be retained between states involved in Josephson tunneling. Letting $\rho_{(i, j)}=\langle n=i|\rho| n=j\rangle$, we can represent the non-zero elements of this reduced density matrix as an 8 component vector:

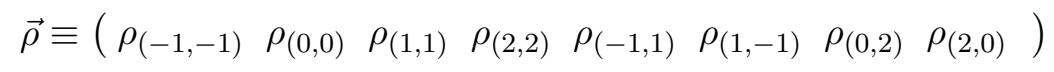

By starting with the von Neumann equation for the evolution of the full density matrix, treating the tunneling Hamiltonian to lowest order in perturbation theory, and then tracing out the fermion degrees of freedom (see, e.g. Ref. [15]), one obtains the an evolution equation for $\rho$. Taking both 
junctions to be on resonance, we have:

$$
M=\left(\begin{array}{cccccccc}
-\Gamma & 0 & 0 & 0 & -i \frac{E_{J}}{2} & i \frac{E_{J}}{2} & 0 & 0 \\
\Gamma & 0 & 0 & 0 & 0 & 0 & -i \frac{E_{J}}{2} & i \frac{E_{J}}{2} \\
0 & 0 & 0 & \Gamma & i \frac{E_{J}}{2} & -i \frac{E_{J}}{2} & 0 & 0 \\
0 & 0 & 0 & -\Gamma & 0 & 0 & i \frac{E_{J}}{2} & -i \frac{E_{J}}{2} \\
-i \frac{E_{J}}{2} & 0 & i \frac{E_{J}}{2} & 0 & -\Gamma / 2 & 0 & 0 & 0 \\
i \frac{E_{J}}{2} & 0 & -i \frac{E_{J}}{2} & 0 & 0 & -\Gamma / 2 & 0 & 0 \\
0 & -i \frac{E_{J}}{2} & 0 & i \frac{E_{J}}{2} & 0 & 0 & -\Gamma / 2 & 0 \\
0 & i \frac{E_{J}}{2} & 0 & -i \frac{E_{J}}{2} & 0 & 0 & 0 & -\Gamma / 2
\end{array}\right)
$$

$E_{J}=g \Delta / 8$ is the standard Ambegaokar-Baratoff value for the Josephson energy emerging from perturbation theory in the tunneling Hamiltonian; $\phi_{L}$ and $\phi_{R}$ are the phases of the superconducting reservoirs. $\Gamma$ is the rate associated with the two quasiparticle transitions occurring the DJQP cycle, and is given by the usual expression for quasiparticle tunneling between two superconductors [16; for simplicity, we assume the two transitions to have equal rates. We have also made a Markov approximation to obtain Eq. (2), which is valid as long we do not probe the evolution of $\rho$ on timescales smaller than $\hbar / E_{C}$.

The zero eigenvector $\vec{\rho}_{0}$ of the evolution matrix $M$ in Eq. (3) corresponds to the stationary value of the reduced density matrix describing the island charge; note that in general, it will have non-zero off-diagonal elements. The diagonal elements of $\rho_{0}$ (which are probabilities) can be obtained from a classical rate equation; one eliminates the off-diagonal elements from Eq. (2) by expressing them in terms of the diagonal elements. Letting $\vec{p}$ be the 4 -vector of these probabilities, we are lead to the rate equation:

$$
\frac{d}{d t} \vec{p}(t)=\left(\begin{array}{cccc}
-\Gamma-\gamma & 0 & \gamma & 0 \\
\Gamma & -\gamma & 0 & \gamma \\
\gamma & 0 & -\gamma & \Gamma \\
0 & \gamma & 0 & -\Gamma-\gamma
\end{array}\right) \vec{p}(t)=0
$$

where $\gamma=E_{J}^{2} / \Gamma$ represents a "rate" for Cooper-pair tunneling. Eq. (5) represents an incoherent model for the DJQP process, and is sufficient for many purposes (e.g., calculating the average current $\langle I\rangle=3 \gamma \Gamma /(4 \gamma+$ $2 \Gamma)$ ). The full stationary value of the density matrix (including off-diagonal 
terms) is given by:

$\vec{\rho}_{0}=\frac{1}{4 \gamma+2 \Gamma}\left(\gamma, \gamma+\Gamma, \gamma+\Gamma, \gamma, i E_{J} e^{-i \phi_{R}},-i E_{J} e^{i \phi_{R}},-i E_{J} e^{-i \phi_{L}}, i E_{J} e^{i \phi_{L}}\right)$

\section{Counting Statistics}

In order to obtain information about the number of electrons that have tunneled through one of the SSET junctions, the density matrix approach of the previous section must be embellished. The standard approach for single electron transistors [15, 4] is to employ a "counter" scheme where one explicitly tracks the dynamics of, say, $m_{L}$, the number of electrons which have tunneled through the left junction of the SSET. The Hilbert space of the SSET is expanded so that each state is now also labelled by a value of $m_{L}$, and the tunnel Hamiltonian is modified so that it raises or lowers this index as appropriate (e.g., by introducing an auxiliary raising operator $F^{\dagger}$ such that $\left.\left[m_{L}, F^{\dagger}\right]=F^{\dagger}\right)$. The resulting equation for the reduced density matrix describing both $n$ and $m_{L}$ is simplified if one Fourier transforms in the latter variable. One finds that $\frac{d}{d t} \vec{\rho}(k)=U^{\dagger} M(k) U \vec{\rho}(k)$, where we have defined:

$$
\begin{gathered}
\rho_{\left(n_{1}, n_{2}\right)}(k)=\left\{\begin{array}{ccccccc}
\sum_{m_{L}} e^{i k m_{L}}\left\langle n_{1}, m_{L}|\rho| n_{2}, m_{L}+2\right\rangle & \text { if } n_{1}=0, n_{2}=2, \\
\sum_{m_{L}} e^{i k m_{L}}\left\langle n_{1}, m_{L}+2|\rho| n_{2}, m_{L}\right\rangle & \text { if } n_{1}=2, n_{2}=0,(7) \\
\sum_{m_{L}} e^{i k m_{L}}\left\langle n_{1}, m_{L}|\rho| n_{2}, m_{L}\right\rangle & \text { otherwise. }
\end{array}\right. \\
M(k)=\left(\begin{array}{cccccccc}
-\Gamma & 0 & 0 & 0 & -i \frac{E_{J}}{2} & i \frac{E_{J}}{2} & 0 & 0 \\
\Gamma e^{i k} & 0 & 0 & 0 & 0 & 0 & -i \frac{E_{J}}{2} & i \frac{E_{J}}{2} \\
0 & 0 & 0 & \Gamma & i \frac{E_{J}}{2} & -i \frac{E_{J}}{2} & 0 & 0 \\
0 & 0 & 0 & -\Gamma & 0 & 0 & i \frac{E_{J}}{2} e^{2 i k} & -i \frac{E_{J}}{2} e^{2 i k} \\
-i \frac{E_{J}}{2} & 0 & i \frac{E_{J}}{2} & 0 & -\Gamma / 2 & 0 & 0 & 0 \\
i \frac{E_{J}}{2} & 0 & -i \frac{E_{J}}{2} & 0 & 0 & -\Gamma / 2 & 0 & 0 \\
0 & -i \frac{E_{J}}{2} & 0 & i \frac{E_{J}}{2} e^{-2 i k} & 0 & 0 & -\Gamma / 2 & 0 \\
0 & i \frac{E_{J}}{2} & 0 & -i \frac{E_{J}}{2} e^{-2 i k} & 0 & 0 & 0 & -\Gamma / 2
\end{array}\right)
\end{gathered}
$$

The probability distribution for $m_{L}$ is thus easily obtained from the characteristic function $p(k, t)$ :

$$
\begin{gathered}
p\left(m_{L}, t\right)=\int_{-\pi}^{\pi} \frac{d k}{2 \pi} e^{-i k m_{L}} p(k, t) \equiv \int_{-\pi}^{\pi} \frac{d k}{2 \pi} e^{-i k m_{L}} \sum_{n} \rho_{(n, n)}(k, t) \\
\vec{\rho}(k, t)=e^{M(k) t} \vec{\rho}(k, t=0)
\end{gathered}
$$


It only remains to specify the initial state of the transistor (i.e. $\vec{\rho}(k, t=0)$ ). The natural choice is to place the transistor $n$ degree of freedom in its stationary state, and choose $m_{L}=0$ with probability one (i.e. $\vec{\rho}(k, t=$ $\left.0)=\vec{\rho}_{0}\right)[15] \cdot p\left(m_{L}, t\right)$ would then represent the stationary probability that $m_{L}$ electrons are transferred through the left junction in a time $t$. While this procedure is perfectly well defined for non-superconducting systems 15, it is problematic in the superconducting case. The stationary state of the SSET has coherence between different charge states- thus, one cannot have both that $m_{L}=0$ and the coherence required in the stationary state. A possible remedy for this problem would be to suppress the off-diagonal elements of the initial density matrix; of course, this procedure needs to be justified. Note that given the linearity of Eq. (10) in the initial density matrix, we can separate incoherent and coherent contributions to $p\left(m_{L}, t\right)$ (i.e. those arising, respectively, from the diagonal and off-diagonal elements of the initial density matrix).

To gain further insight, it is instructive to consider an alternate to the "counter" scheme discussed above. One can also obtain a tunnelled charge distribution for our system by following the general prescription for obtaining full counting statistics discussed in Ref. 9, 10]; we denote this distribution as $p^{\prime}\left(m_{L}, t\right)$. The procedure here involves considering the effects of coupling an ideal measurer of current to the transistor. In practice, a gauge transformation is made to eliminate the interaction with the measurement device, resulting in a phase $e^{i \lambda(t)}$ being attached to the tunnel matrix element $t$ [11]. Using the fact that the perturbative calculation of $\rho$ in the tunneling Hamiltonian has a Keldysh structure (see e.g. Ref. [15]), one finds that $\lambda(t)=\delta+k$ on the forward Keldysh contour, and $\delta-k$ on the backwards Keldysh contour. $\delta$ is a phase which depends on the initial state of the detector; for non-superconducting systems, gauge invariance ensures that it does not play a role. This procedure results in an expression for $p^{\prime}(k, t)$ which is identical in form to Eq. (10), except that now the initial state is uniquely specified as $\vec{\rho}_{0}$, and the evolution matrix $M$ undergoes a $\delta$-dependent unitary transformation which only affects the coherent contribution to $p(k, t)$ :

$$
M \rightarrow V^{\dagger} M V \quad V=\operatorname{diag}\left(1,1,1,1,1,1, e^{-i(k+\delta)}, e^{-i(k-\delta)}\right)
$$

Thus, one has in general phase-dependent counting statistics for our system, meaning that there is a dependence on the phase shift $\delta$ introduced by the measurement. This phase-dependence exists despite the fact that the average current is not sensitive to the phase difference between the two superconducting reservoirs. As discussed in Ref. 10, 12, the results of any charge-counting experiment will now be dependent on the initial state of the detector. We thus see that the ambiguity in defining the initial state of 
the transistor in the "counter" scheme translates here to needing to know the initial state of the detector. Note that the incoherent part of $p(k, t)$ is the same in the two schemes, while the coherent part differs. The process of ignoring the initial coherence of the density matrix in the "counter" scheme corresponds here to assuming an initial detector state which is completely delocalized in the phase $\delta$.

Despite the above caveats, we can determine the counting statistics unambiguously in two limits where the off-diagonal elements of the stationary state $\overrightarrow{\rho_{0}}$ are suppressed- either $\Gamma \gg E_{J}$ or $E_{J} \gg \Gamma$ (c.f. Eq. (6) ). In these limits the coherent contribution to $p(k, t)$ vanishes, implying that the counting statistics are identical in both schemes, and have no dependence on the phase shift $\delta$. Note that in general, the magnitude of the off-diagonal elements of $\rho$ in the stationary state are driven both by the size of $E_{J} / \Gamma$ and by the population asymmetry of the two charge states involved (i.e. $\left.\rho_{02} \propto\left(\rho_{00}-\rho_{22}\right)\right)$. In the limit of large $E_{J}$, it is the lack of population asymmetry which suppresses the off-diagonal elements. In this limit, the symmetric and anti-symmetric superpositions of charge states are equally populated, leading to a vanishing of the off-diagonal matrix elements.

\subsection{THE LIMIT $E_{J} \ll \Gamma$}

In this limit, the SSET effectively gets stuck in the states $n=0$ and $n=1$ waiting for the relatively slow Cooper-pair transitions to occur; we can think of the rate $\gamma$ (c.f. Eq. (5)) as describing effective transitions between $n=0$ and $n=1$ (i.e. $\gamma$ describes both a Cooper-pair event and the subsequent quasiparticle event). We find for the probability distribution $p(m, t) \equiv p\left(m_{L}, t\right)=p\left(m_{R}, t\right)$ (i.e. the junctions are identical) :

$$
p(m, t)=\left\{\begin{aligned}
e^{-\gamma t} \frac{(\gamma t)^{2 l}}{(2 l) !} & \text { if } m=3 l \\
\frac{e^{-\gamma t}}{2} \frac{(\gamma t)^{2 l+1}}{(2 l+1) !} & \text { if } m=3 l+1 \text { or } m=3 l+2 \\
0 & \text { otherwise. }
\end{aligned}\right.
$$

We have neglected terms which are small as $\left(E_{J} / \Gamma\right)^{2}$, and chosen $m=0$ at $t=0$. Again, as the coherent contribution to $p(m, t)$ vanishes to leading order, there is no ambiguity in defining the counting statistics. The statistics can be given a simple interpretation. Each Cooper-pair plus quasiparticle event is described by the rate $\gamma$ and a Poisson distribution. After an even number of $\gamma$ transitions, a multiple of 3 electrons must have been transferred through a junction. However, after an odd number of $\gamma$ transitions, there is an equal probability of having had an extra two electrons transferred (i.e. if we are looking at the left junction, $n=0 \rightarrow 2 \rightarrow 1$ ) or of having a single extra electron transferred (i.e. $n=1 \rightarrow-1 \rightarrow 0$ for the left 
junction). Note that there is an asymmetry here which favours values of $m$ which are an integer multiple of 3 ; this is the analog of the even-odd asymmetry found for the single JQP process [4. Also, note that in this limit the counting statistics are identical if we use the incoherent model of Cooper-pair tunneling described by Eq. (5); coherence plays no role.

\subsection{THE LIMIT $E_{J} \gg \Gamma$}

In this limit, we can think of there being long periods of coherent Josephson oscillations which are interrupted by infrequent quasiparticle transitions; note that we still assume $E_{J} \ll E_{C}=\Delta$, so that it does not effect the energetics of quasiparticle tunneling. Defining the Poisson distribution $\Lambda(l)$ as:

$$
\Lambda(l)=e^{-\Gamma t / 2} \frac{(\Gamma t / 2)^{l}}{l !}
$$

we find up to terms of order $E_{J} / \Gamma$ :

$p(m, t)=\left\{\begin{array}{cl}\frac{1}{4}\left(\Lambda(2 l)\left(3+\frac{\cos \left(E_{J} t\right)}{2^{2 l}}\right)+\Lambda(2 l+1)+\Lambda(2 l-1)\right) & \text { if } m=3 l, \\ \frac{1}{4}\left(\Lambda(2 l+1)+\frac{1}{2} \Lambda(2 l+2)\left(1-\frac{\cos \left(E_{J} t\right)}{2^{2 l+2}}\right)\right) & \text { if } m=3 l+1 \\ \frac{1}{4}\left(\Lambda(2 l+1)+\frac{1}{2} \Lambda(2 l)\left(1-\frac{\cos \left(E_{J} t\right)}{2^{2 l}}\right)\right) & \text { if } m=3 l+2, \\ 0 & \text { otherwise }\end{array}\right.$

where again, we have assumed an initial state with $m=0$ without any ambiguity, as the coherent contribution to $p(m, t)$ is negligible. We can again think of the quasiparticle transitions as effectively being described by a rate $\gamma=\Gamma / 2$ and a Poisson distribution. Now, however, after an even number of transitions (say $2 l$ ), it is not certain that $3 l$ electrons will have been transferred through a junction; rather, due to weakly damped Josephson oscillations, there is also a probability to find $3 l \pm 2$ electrons transferred. Even more remarkably, there are signatures of coherent oscillations in $p(m, t)$, this despite the fact that the initial coherence of the density matrix is irrelevant. As we will see in the next section, the timedependence of the counting statistics has a direct impact on the frequency dependence of the current noise. Note that the oscillation serves to modulate the asymmetry which favours $m$ being a multiple of three. For $\Gamma t \gg 1$, the probability of $m$ being a multiple of 3 is given by:

$$
p(m=3 l) \rightarrow \frac{5+\cos \left(E_{J} t\right)}{8}
$$




\section{Current Noise and Counting Statistics}

In this section, we calculate the frequency dependent current noise $S_{I}(\omega)$ associated with the DJQP process. As is standard, the capacitance of the junctions $C_{L}$ and $C_{R}$ may be used to connect this quantity (which includes the effects of displacement currents) to the noise associated with the tunneling currents in each contact [14]:

$$
S_{I}(\omega)=\frac{C_{L}^{2}}{C_{\Sigma}} S_{L L}(\omega)+\frac{C_{R}^{2}}{C_{\Sigma}} S_{R R}(\omega)+\frac{C_{L} C_{R}}{C_{\Sigma}}\left(S_{L R}(\omega)+S_{R L}(\omega)\right)
$$

where

$$
S_{\alpha \beta}=\lim _{T \rightarrow \infty} \lim _{t_{0} \rightarrow \infty} \int_{-T}^{T} d t e^{i \omega t}\left\langle\left\langle\left\{I_{\alpha}\left(t+t_{0}\right), I_{\beta}\left(t_{0}\right)\right\}\right\rangle\right\rangle .
$$

The order of limits here ensures that we are taking averages with respect to the stationary density matrix of the SSET; note also that we are calculating the classical part of the current noise, which is a symmetric function of frequency. Previous calculations of this quantity for both normal and superconducting transistors have effectively made use of the so-called quantum regression theorem, which involves making an additional Markov approximation beyond that necessary to calculate the reduced density matrix $\rho$ 14, 15, 4]. It turns out that this is not necessary; one can directly connect the particle-current noise correlators to the counting statistics using a formula first derived by MacDonald [17] . Using the definition $I_{\alpha}(t)=\partial_{t} m_{\alpha}(t)$, one can show:

$$
S_{\alpha \beta}(\omega)=2 e^{2} \omega \int_{0}^{\infty} d t(\sin \omega t) \frac{d}{d t}\left\langle\left\langle m_{\alpha}(t) m_{\beta}(t)\right\rangle\right\rangle,
$$

where at $t=0, m_{L}=m_{R}=0$ with certainty, and the system is described by the stationary density matrix $\rho_{0}$. The integral in this expression should be interpreted within the theory of distributions, i.e. $\int_{0}^{\infty} d t \sin \omega t=1 / \omega$. We see from Eq. (18) that the frequency-dependent current noise is directly tied to the time-dependence of the second moment of the tunneled charge distribution. At long times, $\left\langle\left\langle m(t)^{2}\right\rangle\right\rangle \rightarrow f I t / e$, where $f$ is the Fano factor; this leads to the usual expression $S_{\alpha \alpha}(\omega=0)=2 e f I$. A frequency-dependent $S(\omega)$ indicates that $\left\langle\left\langle m(t)^{2}\right\rangle\right\rangle$ deviates from this linear in time behaviour at short times. We thus see that in addition to being useful for calculations, Eq. (18) provides a straightforward way to interpret frequency-dependent current noise in terms of counting statistics. Note that unlike approaches using the quantum regression theorem [14, 4, one does not need to add an ad-hoc term to Eq. (18) to obtain the correct noise in the high-frequency limit. 


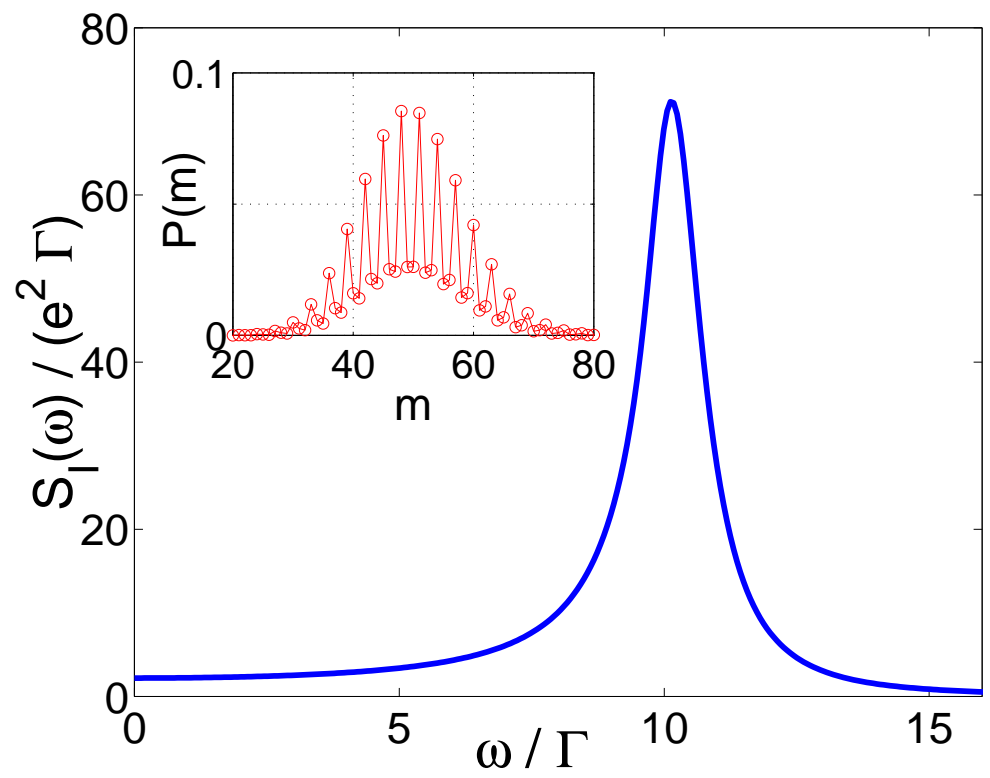

Figure 2. Frequency dependent current noise for the DJQP process at $E_{J}=10 \Gamma$; the large peak at $\omega=E_{J}$ is the result of oscillations in the asymmetry of the tunneled charge distribution. Inset: Distribution of tunneled charge $p(m)$ for the same parameters at $\Gamma t=67$; the asymmetry which favours multiples of 3 is clearly visible.

We apply Eq. (18) to the DJQP process in the limit $E_{J} \gg \Gamma$, where the counting statistics are well-defined and phase-independent. As noted in the previous section, the coherent Josephson oscillations at frequency $E_{J} / \hbar$ modulates the asymmetry in $p(m, t)$ which favours multiples of 3 . This in turn modulates the second moment of the distribution (i.e. the greater the asymmetry, the smaller the width); one finds from Eq. (14):

$$
\left\langle\left\langle m^{2}(t)\right\rangle\right\rangle=\frac{9 \Gamma t}{8}+\frac{9-e^{-\Gamma t}}{8}-\frac{\cos \left(E_{J} t\right)}{2}\left(e^{-\Gamma t / 4}-e^{-3 \Gamma t / 4}\right)
$$

Eq. (18) then implies that there should be a peak in $S_{L L}=S_{R R}$ at $\omega=$ $E_{J}$ of order $e^{2} E_{J}^{2} / \Gamma$, which is much larger than the zero frequency noise $(9 / 4) e^{2} \Gamma$. Shown in Fig. 2 are results obtained for $S_{I}(\omega)$ for $E_{J}=10 \Gamma$, $C_{L}=C_{R}$; a sharp peak is indeed visible. Similar results for the single JQP process were found in Ref. [4. We see here that the peak in the current noise is directly related to the modulation in time of the asymmetry of the counting statistics by coherent Josephson oscillations. 


\section{The Intermediate Regime $E_{J} \simeq \Gamma$}

The noise properties in the regime $E_{J} \simeq \Gamma$ are especially interesting, as it is in this regime where the DJQP process can be used to make a near quantum-limited measurement [5]. In this regime, the off-diagonal elements of the stationary density matrix $\vec{\rho}_{0}$ are by no means small, and thus the coherent, phase-dependent contribution to the counting statistics will play a role. While this in itself is not a problem, the resulting status of $S_{I}(\omega)$ as calculated using Eq. (18) becomes unclear, as the quantity $\partial_{t}\left\langle\left\langle m^{2}(t)\right\rangle\right\rangle$ is phase dependent. The conclusion would thus appear to be that for resonant Cooper-pair tunneling, the finite-frequency current noise is phase dependent, and consequently is sensitive to details of the measurement.

We close by pointing out that the value of the zero frequency noise remains phase independent and unambiguous regardless of the ratio $E_{J} / \Gamma$. At zero frequency, each of $S_{\alpha \beta}$ are equal; thus, we only need to know $\partial_{t}\left\langle\left\langle m^{2}(t)\right\rangle\right\rangle$ in the large time limit. As discussed in Ref. [15], this is determined completely by the lifting of the zero eigenvalue of the evolution matrix $M(k=0)$ in Eq. (3) by non-zero $k$; the contribution to $\left\langle\left\langle m^{2}(t)\right\rangle\right\rangle$ from other eigenmodes of $M$ are damped away in the long time limit. Restricting ourselves to only this "lifted" zero-mode, we have:

$$
p(k, t) \rightarrow e^{\lambda_{0}(k) t}(1+A(k, \delta)),
$$

where $A(k, \delta)$ contains all $k$-dependent terms involving the initial density

matrix, and the $\delta$-dependent eigenvectors (left and right) corresponding to $\lambda_{0}(k) ; A$ vanishes for $k \rightarrow 0$. As:

$$
\left\langle\left\langle m^{2}(t)\right\rangle\right\rangle=-\frac{d^{2}}{d k^{2}} \log p(k, t)
$$

it follows from Eq. (18) that

$$
S_{I}(0) \equiv S_{\alpha \alpha}=2 e^{2}\left(-\left.\frac{d^{2}}{d k^{2}} \lambda_{0}(k)\right|_{k=0}\right)
$$

The phase-dependent term $A$ is thus explicitly seen to play no role. Analyzing the eigenvalue $\lambda_{0}(k)$ of $M(k)$ in Eq. (8), one find for the Fano factor $f[5]$ :

$$
f=\frac{3}{2}\left[1-\frac{6 E_{J}^{2} \Gamma^{2}}{\left(\Gamma^{2}+2 E_{J}^{2}\right)^{2}}\right]
$$

In both the limits $E_{J} \ll \Gamma$ or $E_{J} \gg \Gamma, f \rightarrow 3 / 2$. This can be understood as an effective charge- each Cooper-pair plus quasiparticle transition transfers on average $3 / 2$ electrons per junction. Interestingly, when $E_{J} \sim \Gamma, f$ drops 
below $1 / 2$, reaching a minimum of $3 / 8$ when $E_{J}=\Gamma / \sqrt{2}$. This behaviour is reminiscent of double tunnel junction systems, or of a normal SET; in both cases, there are two rates involved in transport, and the Fano factor reaches a minimum of $1 / 2$ when these rates are equal. The behaviour in such systems can be understood completely classically by the self-averaging that occurs when one sequentially combines two independent Poisson processes. In contrast, the fact that $f$ drops below $1 / 2$ for the DJQP process is a direct consequence of the coherence of Cooper-pair tunneling. To underscore this point, one can calculate $f$ using the incoherent model of Eq. (5). While this procedure yields the correct value of $\langle I\rangle$ and the correct value of $f$ for extreme values of $E_{J} / \Gamma$, it only gives a minimum $f$ of $1 / 2$. One finds:

$$
f_{\text {incoherent }}=\frac{3}{2}\left[1-\frac{2 E_{J}^{2} \Gamma^{2}}{\left(\Gamma^{2}+2 E_{J}^{2}\right)^{2}}\right]=f+\frac{6 E_{J}^{2} \Gamma^{2}}{\left(\Gamma^{2}+2 E_{J}^{2}\right)^{2}}
$$

Note that the incoherent calculation of $f$ is always an overestimate; as expected, coherence between charge states suppresses the zero-frequency current noise, as it tends to make tunnelling events more regular.

\section{Conclusions}

We have studied the counting statistics and finite-frequency shot noise of the DJQP process in a superconducting single electron transistor, attempting to clarify some of the remarkable noise features found in previous studies. In general, the counting statistics are phase-dependent, despite the fact that the average current has no phase dependent. An interesting question remains how this phase dependence impacts the finite frequency noise, given Eq. (18) which directly relates the two quantities.

\section{Acknowledgements}

I am grateful to S. M. Girvin for useful discussions, and to L. Fedichkin for bringing Ref. 17] and Eq. 18 to my attention. This work was supported by the NSA and ARDA under ARO contracts ARO-43387-PH-QC, by the NSF under DMR-0196503 \& DMR-0084501, and by the W.M. Keck Foundation.

\section{References}

1. T. A. Fulton et al., Phys. Rev. Lett. 63, 1307 (1989)

2. D. V. Averin and V. Ya. Aleshkin, JETP Lett. 50, 367 (1989); ibid., Physica B 165 \& 166, 949 (1990). 
3. A. Maasen van den Brink et. al, 85, 459 (1991).

4. M-S. Choi, F. Plastina and R. Fazio, Phys. Rev. Lett. 87, 116601-1 (2001); ibid., cond-mat/0208165

5. A. A. Clerk, S. M. Girvin, A. K. Nguyen and A. D. Stone, Phys. Rev. Lett. 89, 176804 (2002).

6. G. Johansson, cond-mat/0210539

7. Y. Nakamura, Y. A. Pashkin, and J. S. Tsai, Nature 398, 786 (1999).

8. K.W. Lehnert, K. Bladh, L.F. Spietz, D. Gunnarson, D.I. Schuster, P. Delsing, and R.J. Schoelkopf, to appear in Phys. Rev. Lett., 2003.

9. L. S. Levitov, H. W. Lee and G. B. Lesovik, J. Math. Phys., 37, 4845 (1996).

10. Yu. V. Nazarov and M. Kinderman, cond-mat/0107133

11. L. S. Levitov and M. Reznikov, cond-mat/0111057

12. W. Belzig and Yu. V. Nazarov, Phys. Rev. Lett. 87, 067006 (2001).

13. A. Shelankov and J. Rammer, cond-mat/0207343

14. A. N. Korotkov, Phys. Rev. B 49, 10381 (1994).

15. Y. Makhlin et al., Phys. Rev. Lett. 85, 4578 (2000); ibid., Rev. Mod. Phys. 73, 357 (2001).

16. see, e.g., M. Tinkham, Introduction to Superconductivity, (McGraw-Hill, New York, 1996).

17. D. K. C. MacDonald, Rep. Prog. Phys. 12, 56 (1948) 